\title{
An Unusual Low State of the Polar AR UMa
}

\author{
Diana P. Kjurkchieva and Dragomir V. Marchev \\ Dept. of Astronomy, Shumen University, 9700 Shumen, Bulgaria \\ email: d.kyurkchieva@shu-bg.net; d.marchev@shu-bg.net
}

\begin{abstract}
Our photometric CCD observations of the short-period cataclysmic star AR UMa in 2008 during its low state revealed light variability with a bigger period (by around 10\%) and considerably smaller amplitudes than the previous ones. The light curve had a single-wave shape, opposite to the previously observed low states which revealed doubled-humped shape.
\end{abstract}

Keywords. stars: close binaries, cataclysmic variables, individual AR UMa

\section{Introduction}

The very luminous soft X-ray source 1 ES $1113+432$ was optically identified as a nearby (88 pc) short-period variable star AR UMa by Remillard et al. (1994). Old plates showed that it spent most of the time in the low state with $\mathrm{V}=16.5 \mathrm{mag}$ with a sporadic light increase reaching up to $\mathrm{V}=14.5-15 \mathrm{mag}$ (Wenzel 1993).

Remillard et al. (1994) derived a fundamental period of $0.9662 \mathrm{hr}$ and interpreted the light modulations as ellipsoidal variations due to the rotation of a gravitationally distorted M star. As a result, they assumed the orbital period to be twice the fundamental modulation. The observations of Szkody et al. (1999) showed that the light curve of AR UMa at low state had a doubled-humped shape, while during the high state it revealed a single sinusoid variation with amplitude 0.3 mag peaking near phase 0.4 . Howell, Gelino \& Harrison (2001) confirmed doubled-humped structure of the low-state IR light curves of AR UMa and modeled them by beamed cyclotron radiation.

Due to the observed high X-ray luminosity, moderate (2-5\%) circular polarization during the low state (Shakhowskoj \& Havelin 2000), and IUE spectral features, AR UMa was classified as a polar but the confirmation of its extremely strong magnetic field of around 230 MGs was provided by the detection of Zeeman components of $\mathrm{L} \alpha$ and presence of forbidden lines (Schmidt et al. 1996, Gansicke et al. 2001).

The model of AR UMa includes a red secondary filling its Roche lobe and transferring mass to the more massive WD. Its magnetic field diverts the matter from its ballistic trajectory and funnels it along magnetic flux tubes. This confinement of the accreted material results in the formation of accretion columns at the WD's magnetic poles. Mass transfer appears to cease when the binary passes into a low state.

\section{Observations and analysis of the data}

We observed AR UMa during 3 almost consecutive nights in 2008 March (7-10) with the $60 \mathrm{~cm}$ telescope of the Mt. Suhora observatory and CCD camera Apogee ALTA U47UV. The observations were taken in $\mathrm{V}$ filter with exposures $60 \mathrm{sec}$. The standard procedure for reduction of the photometric data was used. For transition from the instrumental system to standard photometric system, we used 3 standard stars AR UMa-1, AR UMa-6, and AR UMa-12 that were constant within 0.005 mag during the all observations. 
The periodogram analysis of all our photometric data performed by the Period04 software (Lenz \& Breger 2005) led to the ephemeris

$$
H J D(M i n)=2454530.618503+0.08850625 * E \text {. }
$$

The obtained period of $2.12415 \mathrm{hr}$ is around $10 \%$ bigger than the known orbital period of $1.932 \mathrm{hr}$.

We phased our photometric data with the ephemeris (2.1). Figure 1 (left) presents the corresponding folded curve. The mean V magnitude of AR UMa during our observations is $16.55 \mathrm{mag}$, which means the polar has been at its low state. The folded curve has a single-wave shape, while the previous light curves of AR UMa in low state have a double-humped shape and bigger amplitude (Fig. 1).

\section{Conclusions}

Our observations of the polar AR UMa at low state revealed several peculiarities:

(a) a period of variability $\sim 10 \%$ bigger than the previously determined value;

(b) considerably smaller amplitude of light variability than those of the previously observed low states;

(c) a single-wave shape of the light curve, in opposition to the doubled-humped light curves observed at the previous low states.

These results show the complex type of the light variability of the polar AR UMa as well as the necessity for additional photometric observations (especially in the optical range) in order to improve this model.
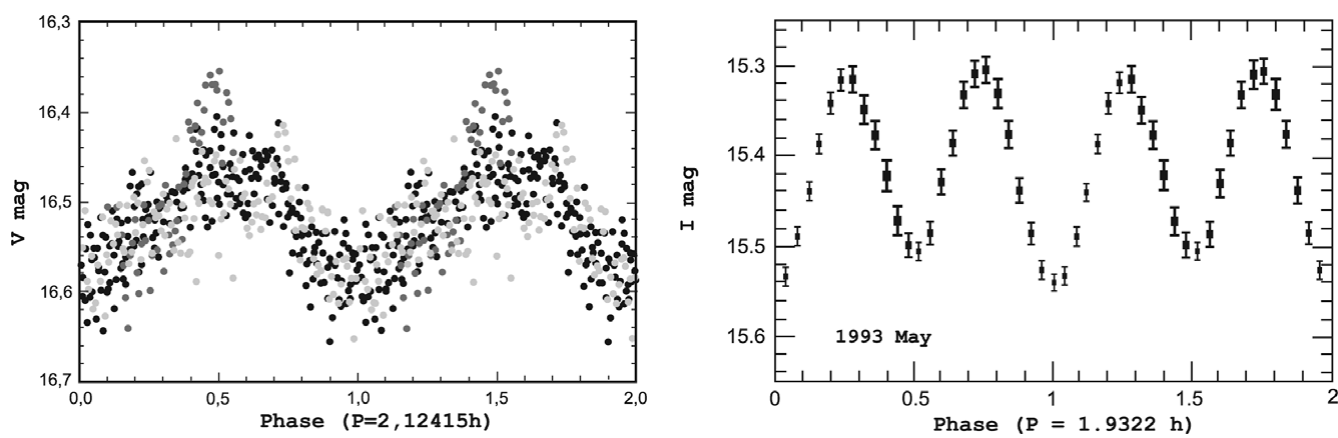

Figure 1. Left: The new V light curve of AR UMa at low state; Right: The old I light curve of AR UMa at low state (Remillard et al. 1994)

\section{Acknowledgements}

The research was supported partly by funds of project DO 02-362 of the Bulgarian Ministry of Education and Science.

\section{References}

Gansicke, B., Schmidt, G., Jordan, S., \& Szkody, P. 2001, ApJ, 555, 380

Howell S., Gelino D., \& Harrison T. 2001, AJ, 121, 482

Lenz P. \& Breger M. 2005, Comm. in Asteroseismology, 146, 53

Remillard, R., Schachter, J., Silber, A., \& Slane, P. 1994, ApJ, 426, 288 
Shakhowskoj N. \& Havelin A. 2000, IBVS, 4858

Schmidt, G., Szkody, P., Smith, P., Silber, A., Tovmassian, G., Hoard, D. W., Gansicke, B. T., \& de Martino, D. 1996, ApJ, 473, 483

Szkody, P., Vennes, S., Schmidt, G., Wagner, R., Fried, R., Shafter, A., \& Fierce, E., 1999, ApJ, 520,841

Wenzel, W. 1993, IBVS, 3890 